\title{
REZENSIONEN
}

\section{Umfangreiches Nachschlagewerk zum politischen System}

Andersen, Uwe und Wichard Woyke (Hrsg.): Handwörterbuch des politischen Systems der Bundesrepublik Deutschland, 6. Auflage, VS-Verlag für Sozialwissenschaften, Wiesbaden 2009, 873 Seiten, € 49,90.

Möchte man sich über das politische System der Bundesrepublik Deutschland informieren, lassen sich unterschiedliche Zugänge wählen. Man kann eines der unzähligen Einführungswerke oder eine der vielen groß angelegten Meistererzählungen zu Rate ziehen, die im vergangenen Jahr anlässlich des sechzigsten Bestehens des Grundgesetzes erschienen sind. Bevorzugt man hingegen einen Überblick, kann man ebenso zu einem nach Stichwörtern sortierten Handwörterbuch über das politische System greifen. Diese Gattung lässt sich als Gebrauchsanweisung für die Politik eines Landes verstehen. In kurzen und prägnanten Beiträgen werden neben den zentralen Institutionen und Akteuren auch die politischen Verfahren und Entscheidungswege beleuchtet. Mit dem Handwörterbuch des politischen Systems der Bundesrepublik Deutschland - nunmehr bereits in der sechsten Auflage erschienen - legen Uwe Andersen und Wichard Woyke ein umfangreiches Nachschlagewerk vor.

Insgesamt umfasst der Band 162 Einzelstichwörter und bietet damit differenzierte Orientierungsmöglichkeiten innerhalb des politischen Systems. Sowohl für den Wissenschaftler als auch für den politisch interessierten Bürger stellt das Buch eine wahre Fundgrube dar, in der man an vielen Stellen gerne verweilt und weiterliest.

Bei näherer Durchsicht fallen jedoch einige Defizite und Schwächen auf, die nicht unerwähnt bleiben sollen. Hat man sich anhand einiger Stichwörter einen ersten Überblick verschafft, erscheint die weiterführende Literatur bei einer Reihe von Einträgen wenig hilfreich. Oftmals werden nur wenige Titel genannt, von denen etliche nicht zum thematisch einschlägigen Kanon gehören. Eine Reihe von Begriffen wird auf denkbar knappem Raum abgehandelt. Beispielsweise werden die für das Verständnis der Bundesrepublik wichtigen Konzepte wie „Pluralismus“ und „Republik“ auf jeweils nur drei Spalten erläutert. Auch die Erklärungen unter dem Stichwort „Nachrichtendienste“ fallen nicht viel länger aus. Kurios erscheint das Schlagwort „Frauen in der Politik - Politik für Frauen“, und auch bei näherer Betrachtung lässt sich diesem Eintrag kaum ein Erkenntnisgewinn abringen. Nach nur einer Seite stößt der Leser schließlich auf die politischen Erinnerungen der ehemaligen Ministerpräsidentin Heide Simonis mit dem schillernden Titel „Unter Männern. Mein Leben in der Politik“ (S. 217), die als weiterführende Literatur empfohlen werden. Erwartungsgemäß werden die Verfassungsorgane des Bundes, also Bundestag, Bundesrat, Bundesregierung, Bundespräsident und Bundesverfassungsgericht einzeln erklärt. Den Eintrag „Bundesversammlung" sucht man hingegen vergeblich.

Zudem werden immer wieder Bewertungen und Einschätzungen vorgenommen, die meinungsgeprägt sind und Widerspruch anregen. Zur deutschen Außenpolitik zu Beginn des 21. Jahrhunderts äußert Wichard Woyke die Auffassung, dass es „[i]n einem sich durch immer größere Interdependenzstrukturen kennzeichnenden internationalen System “ nicht mehr möglich sei, „eine vollkommen nationalstaatlich orientierte Außenpolitik zu betrei- 
ben“ (S. 28). Diese Bedingung außenpolitischen Handelns beschreibt jedoch keineswegs ein Novum, sondern vielmehr ein etabliertes Merkmal deutscher Außenpolitik. Gerade durch den Verzicht einer nationalstaatlich auftrumpfenden Politik hat die Bundesrepublik schon in ihrer Anfangszeit Respekt und Einflussmöglichkeiten hinzugewonnen. Als Motor des europäischen Einigungswerkes sowie durch multilaterale Einbindung ist das deutsche Regierungssystem mittlerweile so weit gezähmt, dass eine rein national gefärbte Politik kaum mehr vorstellbar ist. In seinem Beitrag zur „Demokratie in Deutschland“ konstatiert Dietrich Thränhardt, dass der Nationalstaat in Deutschland lange Zeit keine dominante Leitkategorie gewesen sei und dies zu einer „Scheu vor Staatssymbolen“ (S. 146) geführt habe. So sei auch zu erklären, warum die Ereignisse von 1989/90 nicht als „friedliche Revolution“, sondern als „Wende“ bezeichnet werden. Dieser Zusammenhang bleibt allerdings fraglich, handelt es sich doch vor allem um einen Wahrnehmungsunterschied zwischen Ost und West. Während die Bürger in Ostdeutschland mehrheitlich von Wende sprechen, hat sich bei den Westdeutschen eher das Wort von der Wiedervereinigung durchgesetzt.

Insgesamt fällt auf, dass das Handwörterbuch eine starke Polity-Orientierung aufweist. Institutionen und Politikfelder werden durch unterschiedliche Stichworteinträge abgedeckt, Mechanismen und politische Verfahren bleiben jedoch etwas unterrepräsentiert. Zwar gibt es Einträge zu „Politische Beteiligung“, „Direkte Demokratie“ oder „Pluralismus“. Zentrale Konzepte wie „Politische Steuerung“ oder „Politikverflechtung“ sind hingegen nicht dabei. Für das Verständnis des politischen Systems und seiner Problemlösungsverfahren könnten diese Stichwörter ganz sicher zusätzliche Erkenntnisse liefern. Abgesehen von diesen einschränkenden Befunden handelt es sich bei dem vorliegenden Band um ein nützliches Nachschlagewerk, das man für eine erste Orientierung oder einfach nur noch einmal zur eigenen Wissensauffrischung gut zu Rate ziehen kann. Werden die erwähnten Schwachpunkte in der nächsten Auflage behoben, kann das Handwörterbuch noch ein Stück an Qualität hinzugewinnen.

Helge F. Jani

\section{Populäre Aufbereitungen des Deutschen Bundestages}

Das deutsche Parlament. Mit Beiträgen von Manfred Görtemaker, Everhard Holtmann, Wolfgang Ismayr, Michael S. Cullen und Volker Wagner, Verlag Barbara Budrich, Opladen \& Farmington Hills 2009, 272 Seiten, € 39,90.

Feldkamp, Michael F: Der Deutsche Bundestag. 100 Fragen und Antworten, Nomos Verlagsgesellschaft, Baden-Baden 2009, 208 Seiten, € 19,90.

Im September 2009 feierte der Bundestag den 60. Jahrestag seines Bestehens. Im Gegensatz zum Grundgesetz, das im Mai 60 Jahre alt geworden war, stand der Bundestag allerdings nicht im publizistischen Fokus. Während zur deutschen Verfassung mehrere Bücher veröffentlicht wurden (sogar eines für Kinder) ${ }^{1}$, liegt zum Parlament eine solche Anzahl an

1 Christian Bommarius, Das Grundgesetz. Eine Biographie, Berlin 2009; Christoph Möllers, Das Grundgesetz. Geschichte und Inhalt, München 2009; Marion Detjen / Stephan Detjen / 